\title{
Two-step validation of a Monte Carlo dosimetry framework for general radiology
}

\author{
An Dedulle ${ }^{\mathrm{a}, \mathrm{b}, *}$, Niki Fitousi ${ }^{\mathrm{a}}$, Guozhi Zhang ${ }^{\mathrm{c}}$, Jurgen Jacobs $^{\mathrm{a}}$, Hilde Bosmans ${ }^{\mathrm{b}, \mathrm{c}}$ \\ ${ }^{a}$ Qaelum NV, Gaston Geenslaan 9, 3001 Leuven, Belgium \\ ${ }^{b}$ University of Leuven, Department of Imaging and Pathology, Division of Medical Physics and Quality Assessment, \\ Herestraat 49, 3000 Leuven, Belgium \\ ${ }^{c}$ University Hospitals Leuven, Department of Radiology, Herestraat 49, 3000 Leuven, Belgium
}

\begin{abstract}
The Monte Carlo technique is considered gold standard when it comes to patient-specific dosimetry. Any newly developed Monte Carlo simulation framework, however, has to be carefully calibrated and validated prior to its use. For many researchers this is a tedious work. We propose a two-step validation procedure for our newly built Monte Carlo framework and provide all input data to make it feasible for future related application by the wider community. The validation was at first performed by benchmarking against simulation data available in literature. The American Association of Physicists in Medicine (AAPM) report of task group 195 (case 2) was considered most appropriate for our application. Secondly, the framework was calibrated and validated against experimental measurements for trunk X-ray imaging protocols using a water phantom. The dose results obtained from all simulations and measurements were compared. Our Monte Carlo framework proved to agree with literature data, by showing a maximal difference below $4 \%$ to the AAPM report. The mean difference with the water phantom measurements was around $7 \%$. The statistical uncertainty for clinical applications of the dosimetry model is expected to be within $10 \%$. This makes it reliable for clinical dose calculations in general radiology. Input data and the described procedure allow for the validation of other Monte Carlo frameworks.
\end{abstract}

Keywords: Monte Carlo, Dosimetry, General radiology

\section{Introduction}

A survey from 2014 involving 36 countries from the European Union showed that projection imaging accounted for $87 \%$ of all medical diagnostic examinations [1. The survey showed that the contribution to the total effective dose for diagnostic projection imaging of the population was $22 \%$ [1]. This

\footnotetext{
* Corresponding author

Email addresses: an.dedulle@qaelum.com (An Dedulle), niki.fitousi@qaelum.com (Niki Fitousi), guozhi.zhang@uzleuven.be (Guozhi Zhang), jurgen.jacobs@qaelum.com (Jurgen Jacobs), hilde.bosmans@uzleuven.be (Hilde Bosmans)
} 
highlights the need to track patient dose also in projection imaging, even if there is the tendency to mainly focus on computed tomography (CT) imaging.

A commonly used dosimetric index to estimate the radiation induced risk among the different modalities is the effective dose 2]. A frequently used method to obtain the effective dose from a projection exam is applying conversion factors to the dose-area product $(D A P)$, entrance air kerma at a reference point or (a combination of) other parameters. These conversion factors are obtained for reference patients.

Our ultimate project is situated in the demand for patient specific dosimetry. The world health organization (WHO) estimated that in 2008 over $50 \%$ of both men and women in Europe will be overweight [3], which means that for dosimetric applications over $50 \%$ of the European population will not be appropriately represented by the actual reference body shape. Better dose estimates require new conversion factors based on anatomical models of different weight or body mass index (BMI) groups. Some commercial software packages are commonly used for dose assessment in projection imaging, but these approaches don't represent a realistic anatomical distribution of adipose tissue, soft tissues and bones for the obese or thin patients.

Monte Carlo techniques are often used in dosimetry because of the relative ease and flexibility to calculate or estimate quantities that are difficult to measure, such as absorbed organ doses [4]. Calculations are possible for different modalities, such as general radiology [5, 6], multi slice or cone beam computed tomography (CT) [6, 7, 8, 9, and image-guided radiotherapy [10]. Several Monte Carlo codes are available, such as EGSNRC [11, 12, GEANt4 [13, 14, MCNPX [15] or PEnelope [16, 17.

Any Monte Carlo framework however, has to be carefully calibrated and validated prior to its use. For many researchers the calibration and validation is a tedious work, especially if there is no easy access to a hospital setting. We propose a two-step validation and provide all input data to make it feasible for future applications by the wider community. First our framework was benchmarked through comparison with established codes and secondly we validated against measurements in clinical settings.

\section{Materials and methods}

An in-house developed Monte Carlo framework, which was originally built for cone beam CT [18, 19, was adjusted for dosimetric purposes in projection imaging. The framework was based on EGSNRC (version 4-2.4.0) [12]. Both the Compton and the Rayleigh scattering were included in the photon-electron transport. The XCOM photon cross sections from the National Institute of Standards and Technology (NIST) were used 20. The photon cut-off (PCUT) was set to $0.01 \mathrm{MeV}$. The Kerma Approximation was made on the electrons, i.e. secondary electrons were terminated on their place of 
birth and all their energy was deposited locally, given the short range of electrons in biological tissues at diagnostic energies 2 .

Further input parameters for the Monte Carlo dosimetry framework were the spectrum (implemented as the number of photons per energy bin), the examination geometry and a voxel phantom. During a simulation, the deposited energy from the simulated particles to a predefined region of interest (ROI) is registered and the absorbed dose in the ROI (single voxel or voxel group) can be calculated. The Monte Carlo simulation uncertainty is estimated on a history-by-history basis 21] and quantified as the coefficient of variation (CV), which depends primarily on the number of simulated histories.

We followed a two-step procedure for calibration and validation of the Monte Carlo framework. First it was benchmarked against established data in the literature, by performing the calculations of case 2 of the American Association of Physicists in Medicine (AAPM) Task Group Report 195 [4. Next, the framework was calibrated and validated for clinical protocols of a projection imaging system present at the University Hospitals Leuven.

\subsection{Validation against established data}

Case 2 for projection imaging of the AAPM Task Group Report 195 describes a soft tissue phantom with dimensions of $390 \times 390 \times 200 \mathrm{~mm}^{3}$ (height $\times$ width $\times$ depth) placed at a source to surface distance of $155 \mathrm{~cm}$. The detector dimensions are $39 \times 39 \mathrm{~cm}^{2}$ at a distance of $180 \mathrm{~cm}$ from the source at the center of the field of view ( 0 degree case). The deposited energy per photon was scored in ten regions of interest in the phantom (Fig. 1). The applied spectra were a W/Al $120 \mathrm{kVp}$ spectrum and a monoenergetic beam of $56.4 \mathrm{keV}$, which is the mean energy of the $120 \mathrm{kVp}$ spectrum [4, 22. We repeated the simulation in our framework with exactly the same settings.

\subsection{Calibration and validation in clinical setting}

For the clinical evaluation, a Carestream DRX Evolution device with tube potential ranging from $40-150 \mathrm{kV}$ with $1 \mathrm{kV}$ steps was simulated. The detector has a maximum field size of $35 \times 42 \mathrm{~cm}^{2}$ with a pixel resolution of $139 \times 139 \mu \mathrm{m}^{2}$.

The most common imaging protocols and their related examination settings were extracted with the dose monitoring software Dose (Qaelum NV, Belgium). We focused on the trunk examinations (thorax, abdomen, pelvis, lumbar (L) spine and thoracic (T) spine).

\subsubsection{Ionization chamber dosimetry}

All experimental measurements were performed with a FC65-G farmer-type ionization chamber (SN:1698) coupled to an electrometer (IBA Dosimetry, Germany) (Fig. 2). The ionization chamber was calibrated by IBA's Secondary Standard Dosimetry Laboratory (IBA Dosimetry GmbH, Germany). The air kerma calibration was performed according to the IAEA TRS 277 Code of Practice resulting 

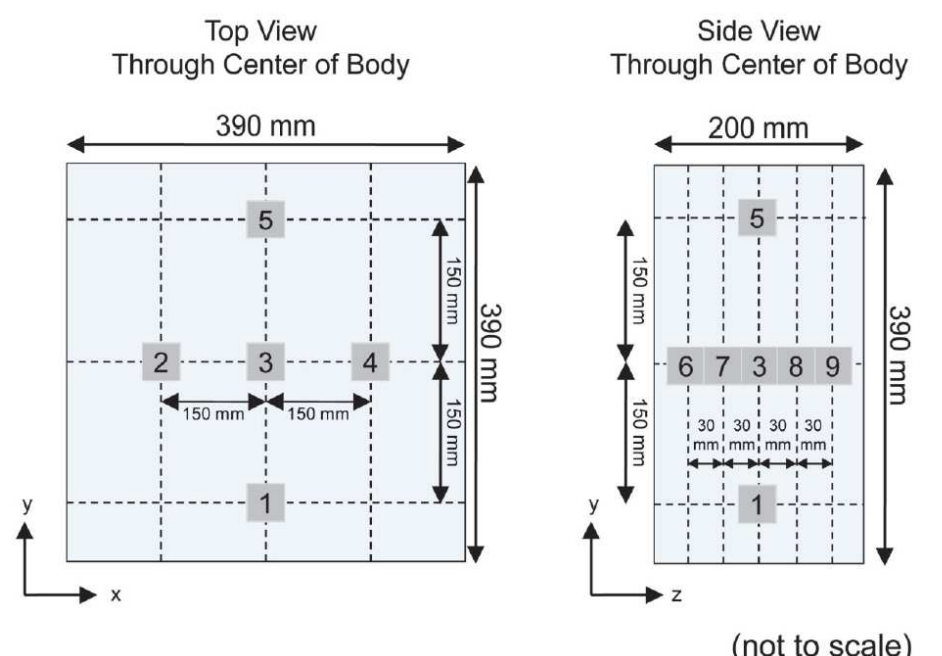

Figure 1: Schematic of the soft tissue phantom and the scoring regions of interest (ROIs) described by 4 . The tenth ROI is the complete phantom.

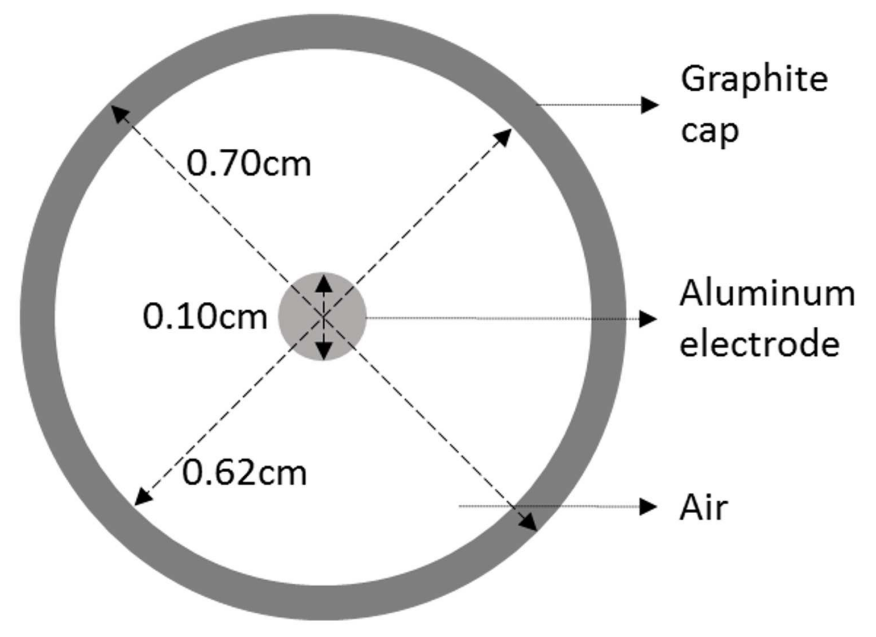

Figure 2: Transversal view of the simulation model of the ionization chamber. The total length of the active part of the ionization chamber is $2.31 \mathrm{~cm}$.

in an air kerma calibration coefficient $\left(N_{K, a}\right)$ of $4.594 \times 10^{7} \mathrm{~Gy} / \mathrm{C}$. For the absorbed dose to water calibration, the procedure was performed according to the IAEA TRS 398 Code of Practice, which resulted in an absorbed dose to water calibration factor $\left(N_{D, w}\right)$ of $4.724 \times 10^{7} \mathrm{~Gy} / \mathrm{C}$.

\subsubsection{Input spectrum}

No bowtie filter is involved in the plain X-ray imaging system. The spectrum is defined by its tube potential and the first half-value layer (HVL). The half-value layer can be determined experimentally with measurements. The ionization chamber was placed free in air at $60 \mathrm{~cm}$ from the tube in a 
collimated beam at the center of the field of view (FOV). The measurement was carried out with a fixed current-time product and by a step-wise addition of thin slabs $(2 \mathrm{~mm})$ of aluminum in the beam path until the measured air kerma was less than half of the initial air kerma. An exponential curve with parameters $a$ and $b$ was fitted to the measurements of the air kerma $(K)$ as a function of the amount of added aluminum $(x)$ :

$$
K=a \mathrm{e}^{b x}
$$

The half-value layer $\left(H V L_{m}\right)$ was then calculated by:

$$
H V L_{m}=\frac{1}{b} \ln \left(\frac{K_{0}}{2 a}\right)
$$

Next, a spectrum with the same half-value layer as the measured value was retrieved. The SPEKTR tool was used to generate the spectra (in $1 \mathrm{keV}$ steps) for a given tube voltage, ripple and filtration [23. Extra layers of aluminum were simulated until the difference between the calculated and measured half-value layer was minimal [24]. This methodology was repeated for all the measured spectra.

\subsubsection{Calibration}

Measured doses are proportional to the tube current-time product (mAs), while the simulated Monte Carlo doses are proportional to the number of simulated histories (hist). A calibration factor is needed to correlate the simulated Monte Carlo dose (mGy / hist) to a measured dose (mGy / mAs).

In the experimental set-up, the ionization chamber (IC) was placed in the center of the field of view and was exposed with a fixed current-time product. In the simulation framework, the geometry of the experimental set-up was implemented together with a model of the ionization chamber (Fig. 2 ). The calibration factor $f_{\text {cal }}$ (hist / mAs) is the ratio of the measured air kerma $K_{m}$ (mGy/mAs) and the simulated Monte Carlo air kerma $K_{M C}(\mathrm{mGy} /$ hist):

$$
f_{c a l}=\frac{K_{m}}{K_{M C}}
$$

The calibration factors were calculated for each trunk protocol. Once the framework is calibrated, the absolute Monte Carlo dose $D_{M C, a b s}(\mathrm{mGy})$ is obtained from multiplication of the Monte Carlo dose per history, $D_{M C}$, with the calibration factor and the current-time product $Q$ of the examination:

$$
D_{M C, a b s}=D_{M C} \times f_{c a l} \times Q
$$

\subsubsection{Validation in a clinical setting}

Validation of our Monte Carlo procedure was performed by measuring absorbed dose deposits in specific configurations for given calibration factors and verifies (relative) differences between simulated 
and measured data. This was carried out for each of the most common trunk protocols to make this study clinically relevant.

For the experimental set-up, a cylindrical, hollow polymethyl methacrylate (PMMA) phantom was filled with water. The center of the field of view (FOV) was at $14.6 \mathrm{~cm}$ from the bottom along the central axis of the phantom. In the AAPM report 195 [4] validation was also done at different locations and depths (Section 2.1), so we opted to validate along the three axes of our set-up (Fig. 3):

- Position A: along the vertical axis of the phantom, $3 \mathrm{~cm}$ above the center of the field of view.

- Position B: along the axis perpendicular to the vertical axis and parallel to the detector, $4 \mathrm{~cm}$ from the center of the phantom.

- Position C: along the detector-tube axis, $6 \mathrm{~cm}$ closer to the detector than the center of the phantom.

Position A was at a depth of $7.6 \mathrm{~cm}$, position B at $6.5 \mathrm{~cm}$ and position $\mathrm{C}$ at $13.6 \mathrm{~cm}$ from the surface of the water phantom, also implying a validation for different attenuation depths.

For each trunk protocol, the dose to water $D_{w, m}$ (mGy) was obtained for the three points in the water phantom. This set-up was implemented in the Monte Carlo framework and the absolute Monte Carlo dose to water $D_{w, a b s}$ (mGy) was calculated according to Equation 4 . The relative error $\left(\left|\Delta_{\text {val }}\right|\right)$ was calculated as:

$$
\left|\Delta_{v a l}\right|=\left|\frac{D_{w, a b s}-D_{w, m}}{D_{w, m}}\right|
$$

\section{Results}

\subsection{Validation against established data}

For both the $120 \mathrm{kVp}$ spectrum and the mono-energetic beam of $56.4 \mathrm{kV}$, the simulation was done with $2.1 \times 10^{8}$ histories and the maximum $\mathrm{CV}$ was $0.3 \%$. The imparted energy per particle for nine scored regions is shown in Fig. 4 along with the mean values from the AAPM 195 report [4]. The mean difference of all scored regions was less than $3 \%$ for both simulated beams (max. $<4 \%$ ).

\subsection{Calibration and validation in a clinical setting}

The commonly used protocols are shown in Table1, different views were included: anterior-posterior (AP), posterior-anterior (PA), lateral (LAT) and towne view (TV). For the chest AP examinations, the source to detector distance is not available since these examinations were taken bedside. We used $100 \mathrm{~cm}$ as source to detector distance for simulations and measurements. 


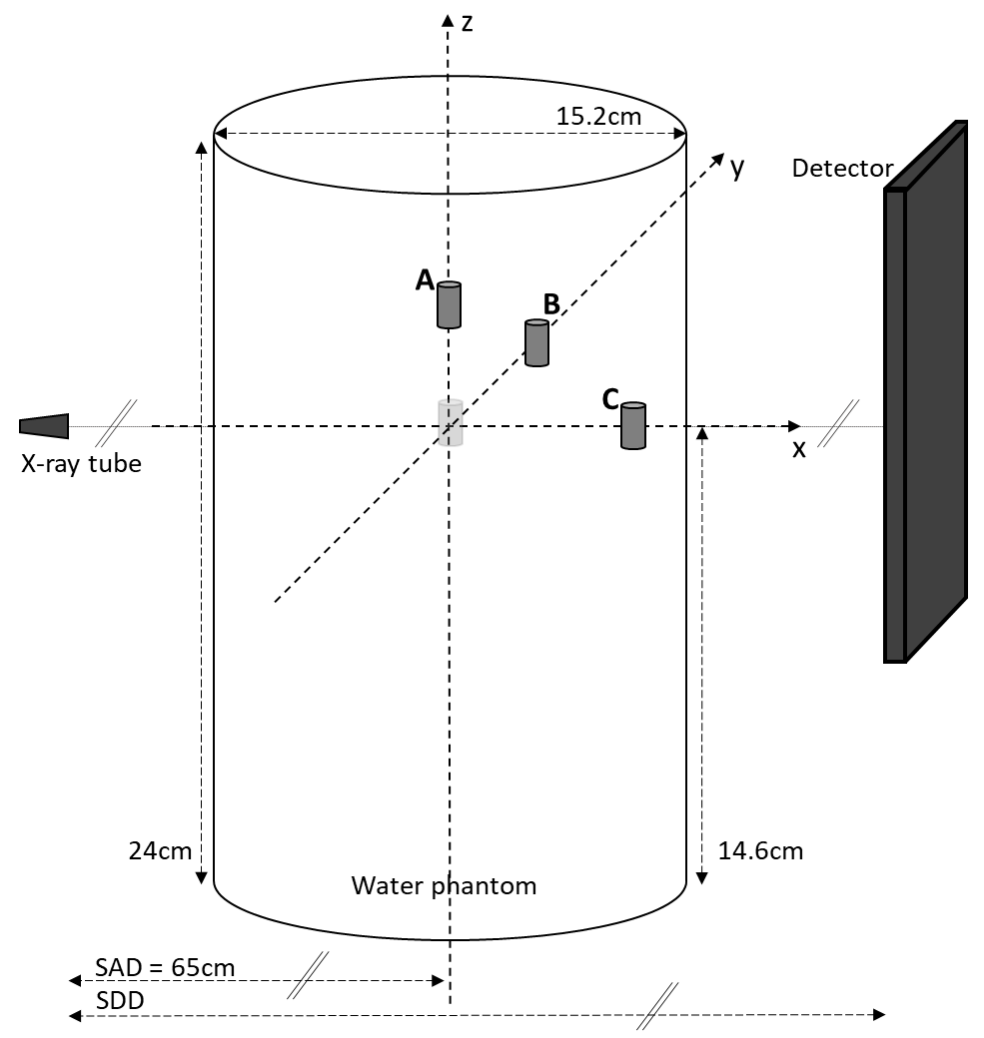

(a) 3-dimensional side-view

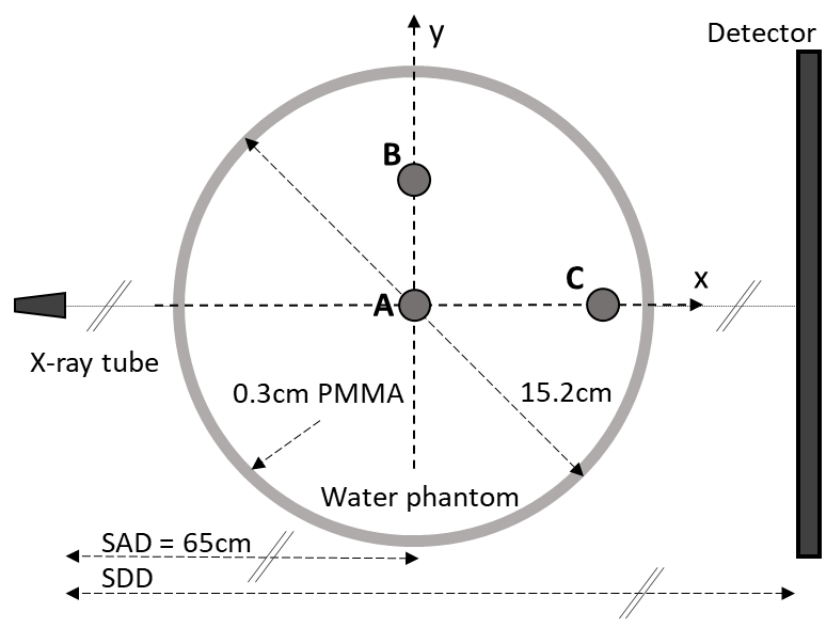

(b) 2-dimensional top-view

Figure 3: The simulation model of the water phantom with spots for the ionization chamber (figure not up to scale). Position A: along the vertical axis, $3 \mathrm{~cm}$ above the center of the field of view. Position B: along the axis perpendicular to the vertical axis and parallel to the detector, $4 \mathrm{~cm}$ from the center of the phantom. Position C: along the detector-tube axis, $6 \mathrm{~cm}$ closer to the detector than the center of the phantom. 


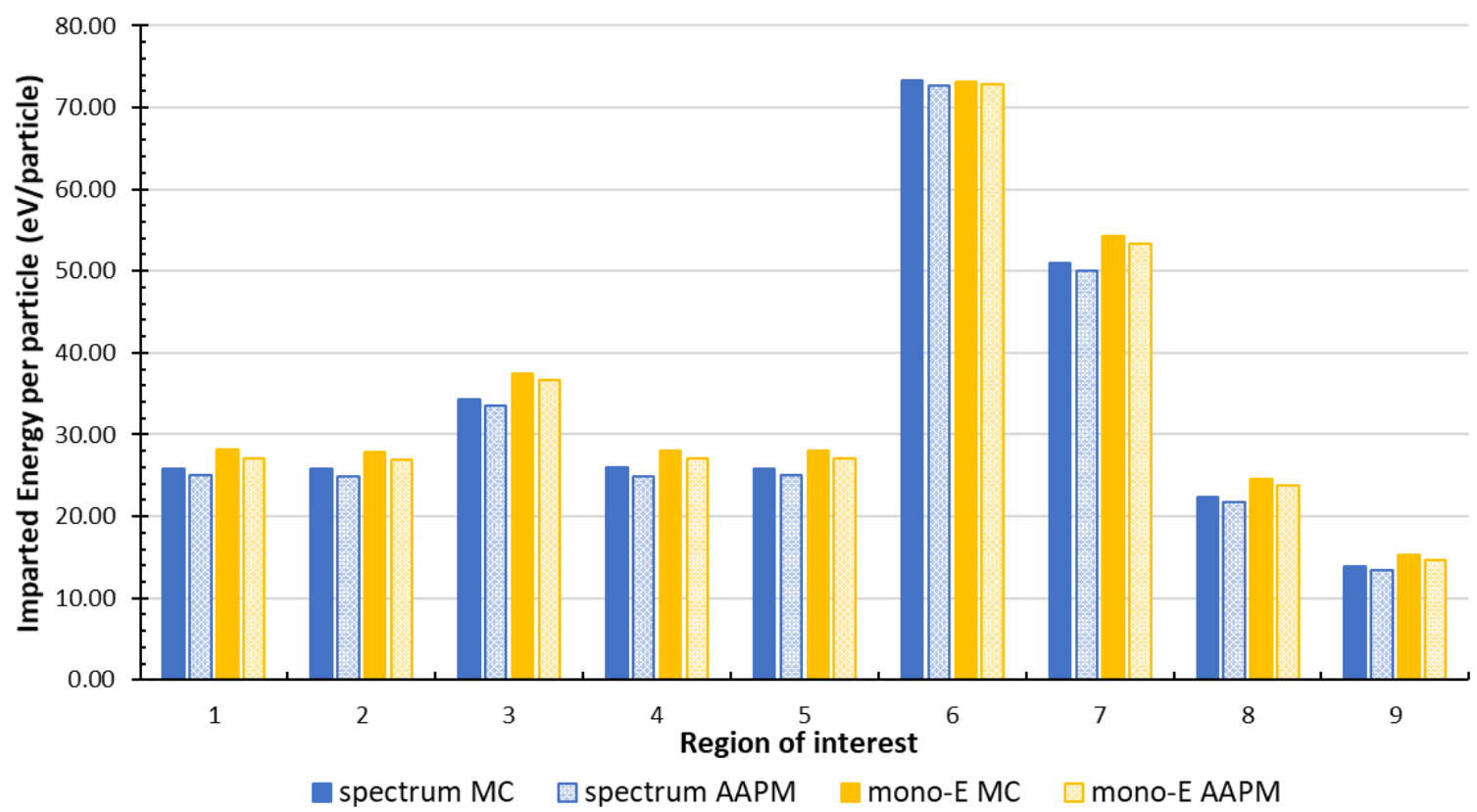

Figure 4: The imparted energy per particle for the 9 ROIs and for both the simulated spectrum and the mono-energetic beam (blue and yellow respectively), the Monte Carlo simulated data is placed next to the mean values from the AAPM 195 report [4.

Table 1: The common trunk protocol settings with the associated tube voltage (U), source to detector distance (SDD), field of view at the detector $\left(\mathrm{A}_{\mathrm{FOV}}[\mathrm{det}]\right)$ and the obtained Monte Carlo calibration factors $\left(f_{\text {cal }}\right)$.

\begin{tabular}{llclll}
\hline Body part - view & $\mathrm{U}(\mathrm{kV})$ & $\mathrm{SDD}(\mathrm{cm})$ & $\mathrm{A}_{\mathrm{FOV}}[\mathrm{det}]\left(\mathrm{cm}^{2}\right)$ & $f_{\text {cal }}\left(\frac{\text { hist }}{\mathrm{mAs}}\right)$ \\
\hline Abdomen & AP & 75 & 140 & $35 \times 42$ & $6.13 \times 10^{10}$ \\
Abdomen & LAT & 85 & 140 & $35 \times 42$ & $8.88 \times 10^{10}$ \\
Chest & AP & 105 & $100^{*}$ & $42 \times 35$ & $2.73 \times 10^{11}$ \\
Chest & LAT & 125 & 179 & $35 \times 42$ & $1.36 \times 10^{11}$ \\
Chest & PA & 120 & 179 & $42 \times 35$ & $1.27 \times 10^{11}$ \\
Pelvis & TV & 75 & 140 & $42 \times 34$ & $6.32 \times 10^{10}$ \\
L Spine & AP & 85 & 115 & $22 \times 41$ & $8.08 \times 10^{10}$ \\
L Spine & LAT & 95 & 115 & $24 \times 41$ & $1.08 \times 10^{11}$ \\
T Spine & AP & 75 & 115 & $20 \times 41$ & $4.97 \times 10^{10}$ \\
T Spine & LAT & 75 & 115 & $24 \times 41$ & $6.14 \times 10^{10}$ \\
\hline
\end{tabular}

* bedside examination (SDD not available), $100 \mathrm{~cm}$ was used 


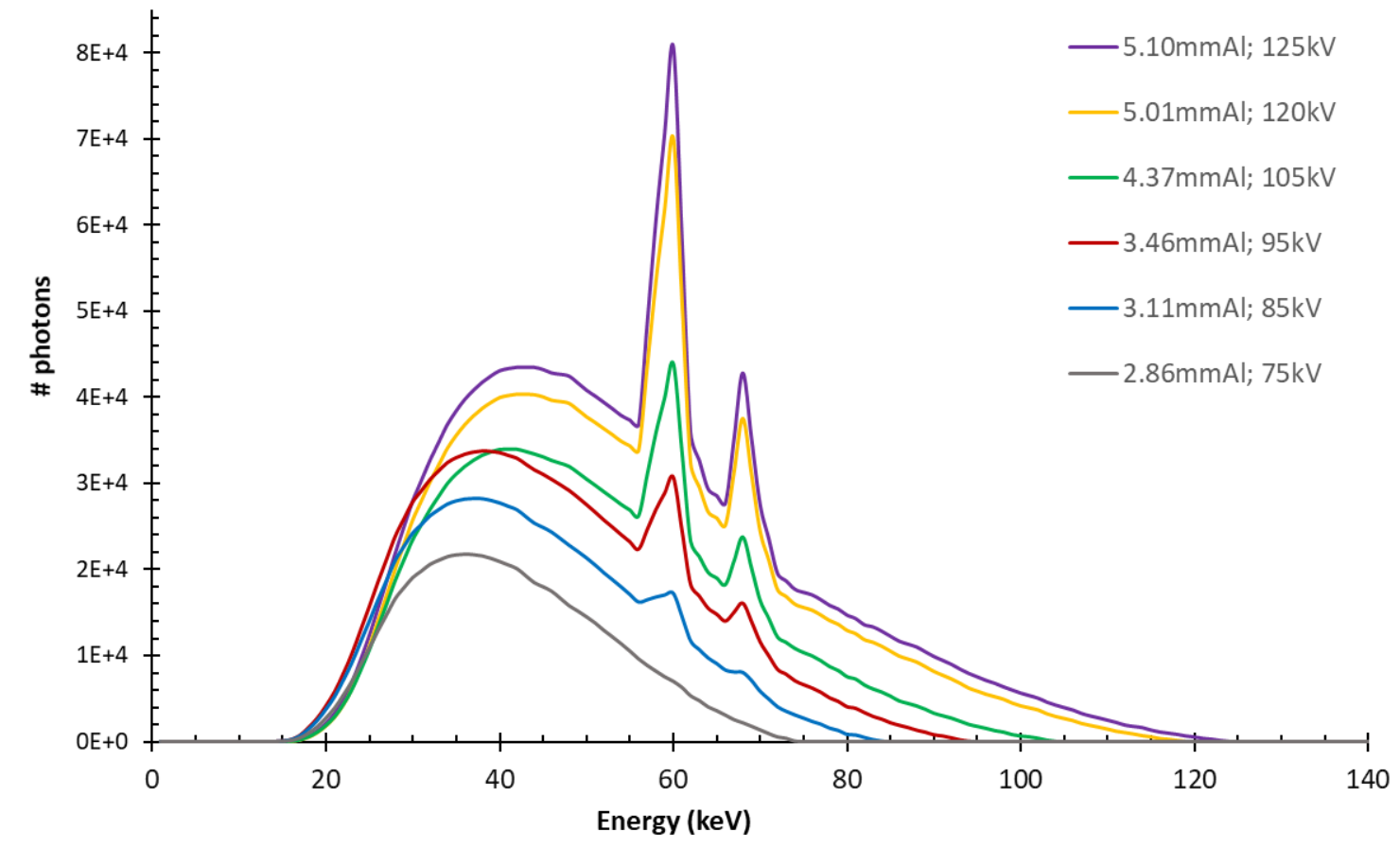

Figure 5: The spectra of the Carestream DRX Evolution for the different tube potentials along with the measured half-value layer.

\subsubsection{Spectrum}

The measured half-value layers of the Carestream DRX Evolution device ranged from $2.86 \mathrm{mmAl}$ to $5.10 \mathrm{mmAl}$ (Fig. 5). As no extra filtration was added for the specified protocols (Table 1), the filtration only included inherent and tube filtration.

\subsubsection{Calibration}

The calibration factors $\left(f_{\text {cal }}\right)$ for each protocol are shown in Table 1 . The field of view at the detector $\left(A_{F O V}[d e t]\right)$ was recalculated to the level of the ionization chamber $\left(A_{F O V}[I C]\right)($ at $60 \mathrm{~cm}$ from the source):

$$
A_{F O V}[I C]=A_{F O V}[\operatorname{det}]\left(\frac{60 \mathrm{~cm}}{S D D}\right)^{2}
$$

While the ionization chamber measurements in air are not affected when the distance and tube output is kept constant, the situation is different for the simulated doses: to speed up our calculations, all histories were confined to the field of view. With the initial number of histories kept constant, this means that the simulated dose will scale with the field of view. So the calibration was repeated for several fields of view. For all spectra the calibration factor was proportional to the field of view size 


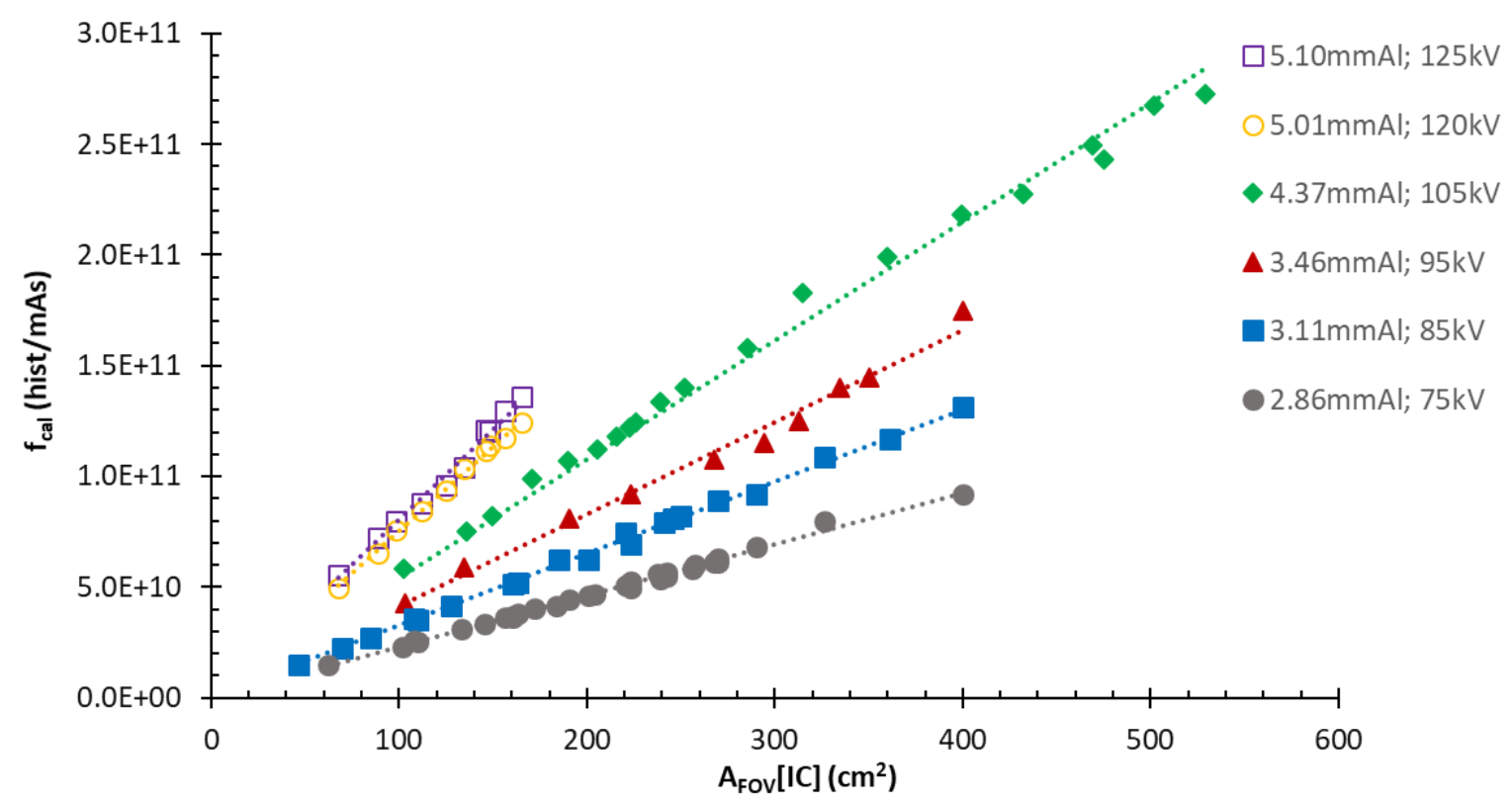

Figure 6: The calibration factors $\left(f_{\text {cal }}\right)$ as a function of the field of view at the position of the ionization chamber $\left(A_{F O V}[I C]\right)$ for the different tube voltages (with related half-value layer). Parameters of the curve fit are presented in Equation A.1 of Appendix A

and the regression curve (through the origin) was calculated with the least square method (Fig. 6). All six curves had an $r^{2} \geq 0.99$.

The slopes of the calibration curves were plotted against the tube voltage (Fig. 7a) and measured half-value layer (Fig. 7b). For both curves there was an excellent exponential correlation (respectively $r^{2}=0.99$ and $\left.r^{2}=0.95\right)$.

The simulations were carried out with $2.1 \times 10^{9}$ histories. The average CV was $3.4 \%$ (max. $5.8 \%$ ) for the simulated air kerma of the chamber. The experimental measurement, on the other hand, was performed 5 times for each tube potential, resulting in a CV less than $2.0 \%$, which is due to the uncertainty of the calibration of the chamber itself as well as the system error in the experiment. Following Equation 3 , the statistical uncertainty associated with the calibration factor was estimated to be around $4 \%$.

\subsubsection{Data for validation}

The doses for positions A and B were quite similar, since their depth from the tube direction in the phantom was similar, receptively $7.6 \mathrm{~cm}$ and $6.5 \mathrm{~cm}$, however their height in the phantom was different. The measured dose in position $\mathrm{C}$, at a depth of $13.6 \mathrm{~cm}$, was lower due to more shielding of the water. The expected increase in measured dose with increasing tube voltage and the related half-value layer was also observed (Fig. 8). 


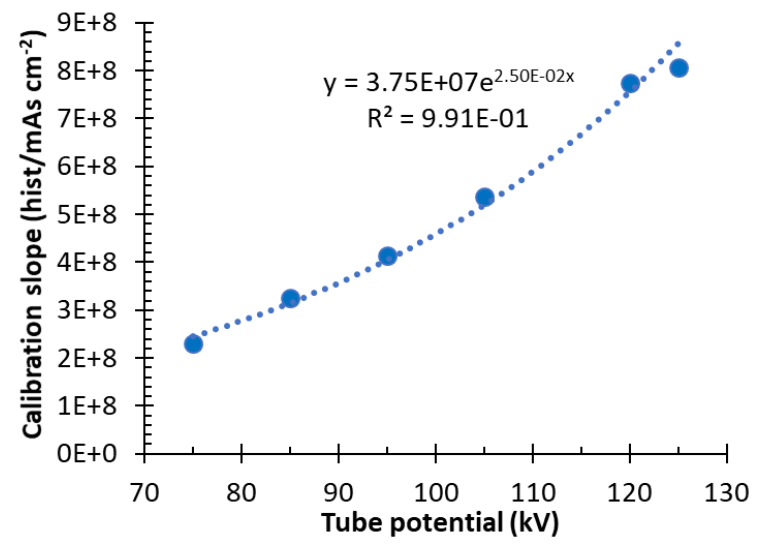

(a)

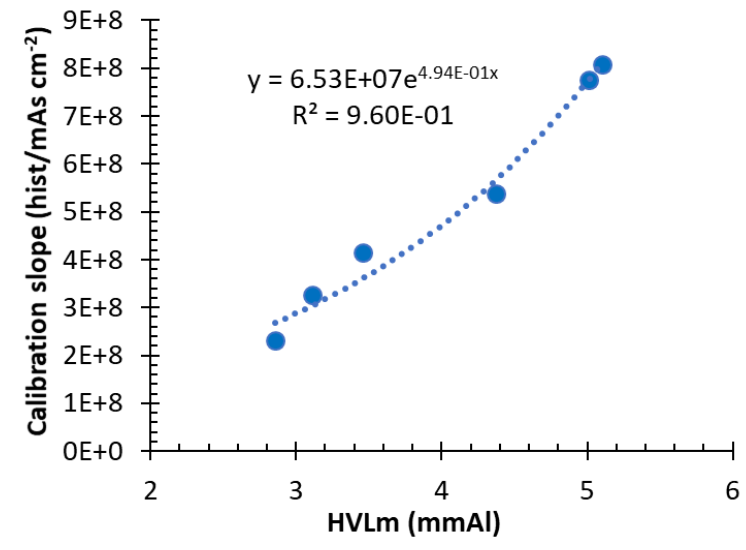

(b)

Figure 7: Slope of the Monte Carlo calibration factor fitted as a function of (a) the tube potential and (b) the measured half-value layer $\left(H V L_{m}\right)$.

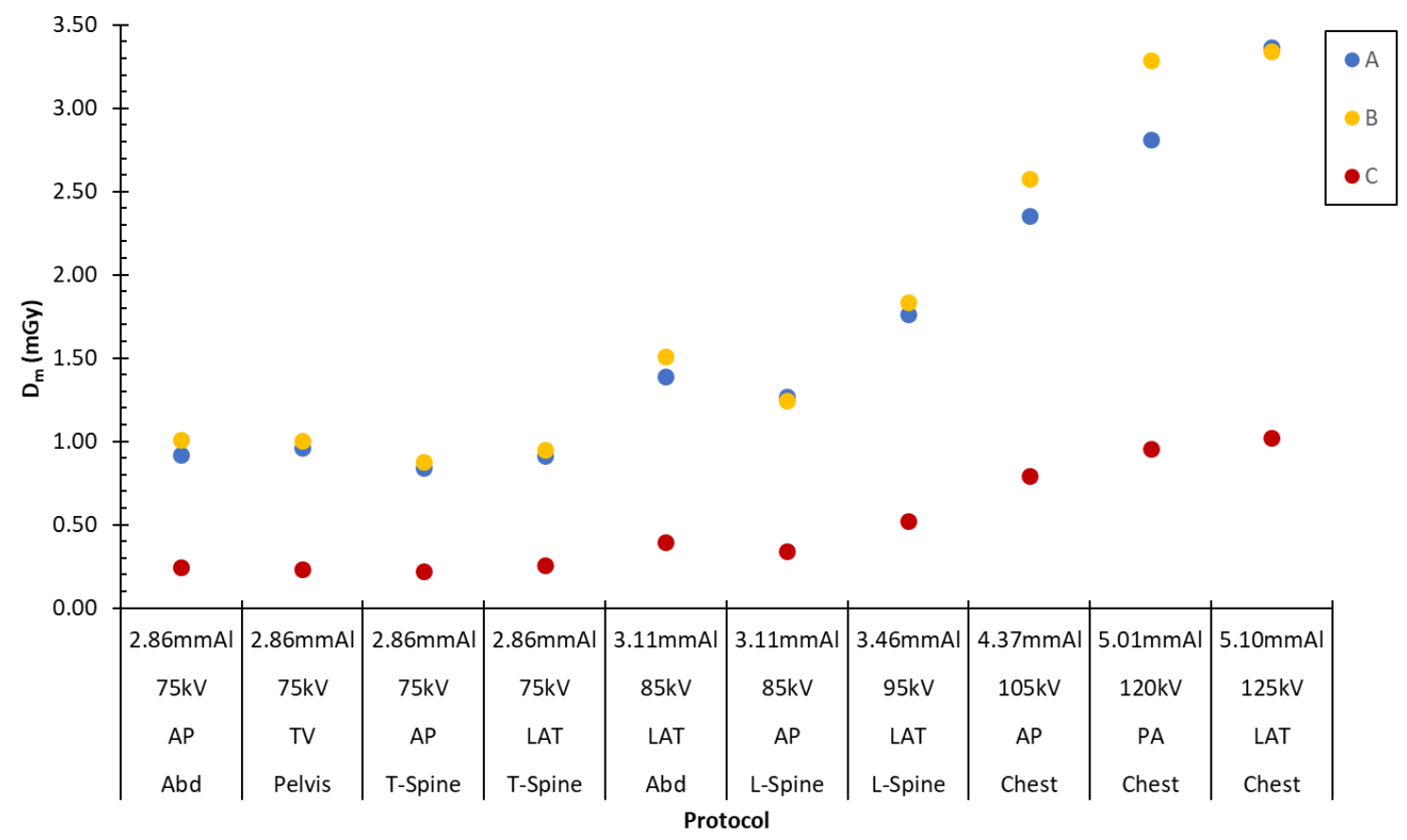

Figure 8: Measured doses $\left(D_{m}\right)$ during the validation of the trunk protocols for three positions $(\mathrm{A}, \mathrm{B}, \mathrm{C})$.

\subsubsection{Validation of a clinical setting}

The mean difference of all validation values was $7 \%$ (max. $16 \%$ ), the differences $\left(\left|\Delta_{\text {val }}\right|\right)$ for all the protocols are shown in Fig. 9. The different positions were analyzed separately too. For positions A and $\mathrm{B}$ the mean difference was $6 \%$, while the largest differences were observed for position $\mathrm{C}$, with a mean of $10 \%$. For all validation points ( $10 \times 3$ positions), 22 of the points were within $10 \%$ and 29 


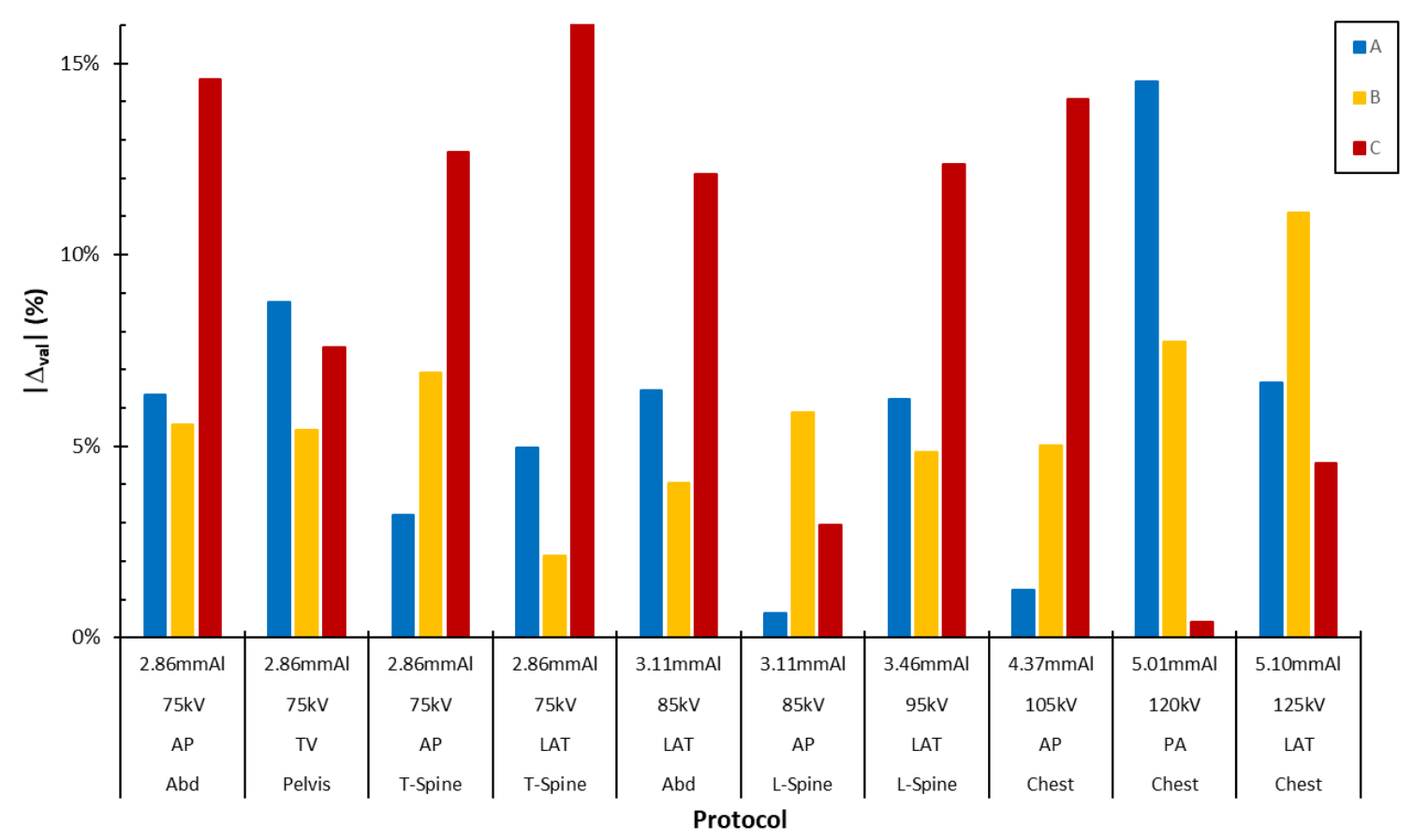

Figure 9: The difference $\left(\left|\Delta_{v a l}\right|\right)$ between the simulated and measured dose in water of the trunk protocols for three positions (A, B, C).

points within $15 \%$, with mostly a small underestimation in the simulated dose for the tube potentials below $120 \mathrm{kV}$ and an overestimation for the higher ones.

For the calculation of the dose in water, the simulation was performed with $2.0 \times 10^{8}$ histories and the average $\mathrm{CV}$ for different locations was found to be $0.8 \%$ (max. 1.5\%). As mentioned above, the statistical uncertainty of the Monte Carlo calibration factor $\left(f_{\text {cal }}\right)$ was around $4 \%$, which is passed on through the absolute dose estimates (Equation 4). The overall statistical uncertainty, therefore, is roughly estimated to be $5 \%$ for the dose calculation model in our Monte Carlo framework, given that the simulations are always associated with a similar CV as in the simulation of the water phantom.

\section{Discussion}

An EGSNRC based Monte Carlo framework was at first successfully validated by benchmarking the simulated data against dosimetry data for projection imaging from the report of the AAPM Task Group 195 (study case 2 of the report). The mean difference between the simulation and the literature data was $3 \%$. Secondly the framework was calibrated and validated in a clinical setting for ten trunk protocols. The average difference between simulated doses and measurements was $7 \%$.

There can be several reasons for the work-out of a new Monte Carlo framework. In our case, it was 
triggered by envisioned future dosimetric requests: commercially available packages have great practical value in a multitude of applications, but there are some limitations, such as the limited flexibility in the type and number of models that are used. As an example, PCXMC (STUK, Finland) 25] has only limited options for mathematical phantoms (5 paediatric and 1 adult), which are all hermaphrodite [26]. While these mathematical models are scalable for weight and height [25], the difference in human anatomy between particular groups of patients (like normal weight versus obese) cannot be realistically modelled [27. Recent literature confirms that using mathematical phantoms will lead to significant (organ) dose differences compared to patient specific voxel models [28] 29]. Additionally, an in-house developed platform allows also more control of the examination geometry, such as real collimation and beam positioning. Another fundamental reason to use an in-house built framework is to hold control over all physics parameters, such as the photon and electron cut-off conditions or the cross section database. More physics groups may be in a similar situation. They would not only have to develop a framework but also calibrate and validate it. For this task, we have developed a procedure that we have documented now in full and for which all input data can be found in (electronic) attachments 30.

A two-step validation was chosen, at first by benchmarking against data from literature and secondly a calibration and validation using dosimetric data of real clinical settings.

The first step uses literature data, but this is scarce and a relevant geometry and photon energy is required (e.g. excluding radiotherapy). The report of AAPM task group 195 222] was used for this step of validation as it has a good and clear description of the used geometry, spectrum and material compositions. The complete data set of their simulations, used spectra and material compositions are on-line available and condition sine qua non for its use as reference [31.

For the second step, the Monte Carlo framework was first calibrated and then validated in a clinical setting. The calibration factor has a proportional relationship with the field of view for the different spectra (Fig. 6), which could be used to inter- and extrapolate to other fields of view. The slopes of these curves are plotted against their tube voltage and half-value layer (Fig. 7). From the associated exponential equation, we could calculate the slope $\left(S_{U}\right)$ of another tube voltage $(U)$. This allows for an estimate of the calibration factor $\left(f_{c a l, U}\right)$ for a given field of view at the detector $\left(A_{F O V}[d e t]\right)$, source to detector distance $(S D D)$ and tube voltage $(U)$ :

$$
\begin{aligned}
S_{U} & =3.75 \times 10^{7} \frac{\text { hist }}{\mathrm{mAscm} 2} \times \mathrm{e}^{2.50 \times 10^{-2} \mathrm{kV}^{-1} U} \\
f_{c a l, U} & =S_{U} \times A_{F O V}[\text { det }]\left(\frac{60 \mathrm{~cm}}{S D D}\right)^{2}
\end{aligned}
$$

Alternative to the tube voltage, the half-value layer $(H V L)$ could be used to calculate the slope $\left(S_{H}\right)$ and the calibration factor $\left(f_{c a l, H}\right)$ : 


$$
\begin{aligned}
S_{H} & =6.64 \times 10^{7} \frac{\text { hist }}{\mathrm{mAscm} 2} \times \mathrm{e}^{4.89 \times 10^{-1} \mathrm{mmAl}^{-1} H V L} \\
f_{c a l, H} & =S_{H} \times A_{F O V}[\text { det }]\left(\frac{60 \mathrm{~cm}}{S D D}\right)^{2}
\end{aligned}
$$

Roughly estimated, the statistical uncertainty of the calibration was around $4 \%$. Our measurements could be repeated in a calibration laboratory to reduce the intrinsic errors. For the subsequent uncertainty of any absolute Monte Carlo dose, the uncertainty of the dosimetric simulation has to be added.

A first challenge when performing the calibration and validation for projection images is the limited information on the positioning of the modelled structures (ionization chamber and water phantom) in the direction between tube and detector due to the two dimensional nature of these images. In CT experiments, a CT scan could be performed for verification and generation of a voxel model [18. During the measurements, the distance to the tube had to be very carefully determined which made the process time consuming. For the simulations we always positioned the chamber exactly at the predefined positions and thus assumed an ideal geometry. Small misplacements however may be reflected in the validation errors. Another limitation is the description of the spectrum and (inherent) filtration: the anode heel effect is not taken into account. Additionally, there is a small difference in path length when a photon crosses the filter centrally or peripherally, but notwithstanding this fact, the central thickness of the filter was used in all cases. Both phenomena could be further investigated and might improve the framework $\underline{32}$, but they will also make the spectra measurements cumbersome and will increase the computational time.

Other groups have performed calibration and/or validation with thermoluminescence dosemeters (TLDs). The use of an ionization chamber avoids the problem of the angular dependency and energy response of TLD dosimetry [33. In addition, TLD measurements are known to be time consuming and reliable positioning is difficult. For validation of CT imaging the CTDI body and/or head phantom is used, and reported validation values from Li et al. (2011) 34 ranged from $-4.8 \%$ to $2.2 \%$. Jarry et al. (2003) 35 reported values within $8 \%$ and $9 \%$ for the head and body phantom respectively. These validation values are a bit lower than in this work, but in contrary to CT imaging, we did not have information on the exact location of the ionization chamber. Due to the number of influencing parameters and the complexity of Monte Carlo dose simulations, differences within $20 \%$ have been considered as good approximations [36. Since all our validation values were within 15\%, except for one value which was $16 \%$, we can consider the framework, including the spectra and calibration factors, validated.

The statistical uncertainty of our Monte Carlo framework was estimated around $5 \%$ for the dose calculation model of the clinical validation. However for organ dose calculations, the coefficient of 
variation $(\mathrm{CV})$ of small organs or organs outside the field of view might be higher, but the CV will still be below $5 \%$. So for organ dose calculations, the overall statistical uncertainty of our simulation framework will be below 10\%. According to the International Atomic Energy Agency (IAEA), an accuracy of $20 \%$ is sufficient for estimating the absolute risk from radiology examinations of adults [37.

\section{Conclusion}

A two-step procedure was proposed for calibration and validation of a general projection Monte Carlo framework for dosimetric applications. Application of the methodology to our in-house Monte Carlo framework, showed the framework to be ready for patient specific dosimetry applications. Additionally all data of the set-up and measurements are provided (electronically [30]), so that other groups could use it to validate their Monte Carlo framework for dosimetric trunk examinations.

\section{Acknowledgements}

The work was conducted in cooperation with Qaelum and the medical physics experts of the University Hospital of Leuven.

Funding: This work was supported by the Flanders Innovation \& Entrepreneurship agency [Grant No. HBC.2016.0233].

\section{Disclosure statement}

An Dedulle has a PhD grant from the Flanders Innovation \& Entrepreneurship agency [Grant No.

HBC.2016.0233] and works as a PhD researcher for Qaelum NV in cooperation with the University of Leuven.

Niki Fitousi is head of research in Qaelum NV.

Guozhi Zhang no disclosure.

Jurgen Jacobs is CEO and co-founder of Qaelum NV.

Hilde Bosmans is board member and co-founder of Qaelum NV.

\section{References}

[1] European Union, Medical radiation exposure of the european population (part1/2), radiation protection n180, Publications Office of the European Union. 
[2] International Commission on Radiological Protection, The 2007 recommendations of the international commission on radiological protection. icrp publication 103, Annals of the ICRP 37 (2-4) (2007) 1-332.

[3] World Health Organisation (Europe), Obestity - data and statistics, http://www.euro.who. int/en/health-topics/noncommunicable-diseases/obesity/data-and-statistics (Last accessed on 22/01/2018).

[4] American Association of Physicists in Medicine, Monte carlo reference data sets for imaging research, aamp task group 195, American Association of Physicists in Medicine.

[5] J. C. Yanch, R. H. Behrman, M. J. Hendricks, J. H. McCall, Increased radiation dose to overweight and obese patients from radiographic examinations, Radiology 252 (1) (2009) 128-39, pMID: 19403846. doi:https://doi.org/10.1148/radiol.2521080141.

[6] Y. Zhang, X. Li, W. P. Segars, E. Samei, Comparison of patient specific dose metrics between chest radiography, tomosynthesis, and ct for adult patients of wide ranging body habitus, Medical Physics 41 (2) (2014) 023901, 023901. doi:http://dx.doi.org/10.1118/1.4859315

[7] C. Lee, K. P. Kim, D. Long, R. Fisher, C. Tien, S. L. Simon, et al., Organ doses for reference adult male and female undergoing computed tomography estimated by monte carlo simulations, Medical Physics 38 (3) (2011) 1196-206. doi:https://doi.org/10.1118/1.3544658.

[8] A. Ding, Y. Gao, H. Liu, P. F. Caracappa, D. J. Long, W. E. Bolch, et al., Virtualdose : a software for reporting organ doses from ct for adult and pediatric patients, Physics in Medicine and Biology 60 (14) (2015) 5601-25. doi:https://doi.org/10.1088/0031-9155/60/14/5601.

[9] A. Stratis, G. Zhang, X. Lopez-Rendon, C. Politis, R. Hermans, R. Jacobs, et al., Two examples of indication specific radiation dose calculations in dental $\{\mathrm{CBCT}\}$ and multidetector $\{\mathrm{CT}\}$ scanners, Physica Medica 41 (2017) 71-7. doi:https://doi.org/10.1016/j.ejmp.2017.03.027.

[10] G. X. Ding, D. M. Duggan, C. W. Coffey, Accurate patient dosimetry of kilovoltage cone-beam ct in radiation therapy, Medical physics 35 (3) (2008) 1135-44. doi:https://doi.org/10.1118/ 1.2839096 .

[11] W. Nelson, N. Hirayama, D. Rogers, The egs4 codesystem, SLAC Report No. 265 (Stanford Linear Accelerator Center).

[12] I. Kawrakow, E. Mainegra-Hing, D. Rogers, F. Tessier, B. Walters, The egsnrc code system: Monte carlo simulation of electron and photon transport. nrcc report pirs-701., NRCC Report PIRS-701. 
[13] S. Agostinelli, J. Allison, K. Amako, J. Apostolakis, H. Araujo, P. Arce, et al., Geant4a simulation toolkit, Nuclear Instruments and Methods in Physics Research Section A: Accelerators, Spectrometers, Detectors and Associated Equipment 506 (3) (2003) 250 - 303. doi:https: //doi.org/10.1016/S0168-9002(03)01368-8.

[14] J. Allison, K. Amako, J. Apostolakis, H. Araujo, P. A. Dubois, M. Asai, et al., Geant4 developments and applications, IEEE Transactions on Nuclear Science 53 (1) (2006) 270 - 8. doi:https://doi.org/10.1109/TNS.2006.869826.

[15] L. Waters, MCNPX User's manual, Los Alamos National Laboratory, briesmeister, judith f. Edition (September 2002).

[16] J. Sempau, E. Acosta, J. Baro, J. Fernndez-Varea, F. Salvat, An algorithm for monte carlo simulation of coupled electron-photon transport, Nuclear Instruments and Methods in Physics Research Section B: Beam Interactions with Materials and Atoms 132 (3) (1997) 377-90. doi: http://dx.doi.org/10.1016/S0168-583X(97)00414-X.

[17] F. Salvat, J. M. Fernndez-Varea, J. Sempau, PENELOPE-2008: A Code System for Monte Carlo Simulation of Electron and Photon Transport, NUCLEAR ENERGY AGENCY, 2008.

[18] G. Zhang, R. Pauwels, N. Marshall, E. Shaheen, J. Nuyts, R. Jacobs, et al., Development and validation of a hybrid simulation technique for cone beam ct: application to an oral imaging system, Physics in Medicine and Biology 56 (18) (2011) 5823-43. doi:https://doi.org/10. 1088/0031-9155/56/18/004

[19] G. Zhang, N. Marshall, R. Bogaerts, R. Jacobs, H. Bosmans, Monte carlo modeling for dose assessment in cone beam ct for oral and maxillofacial applications, Medical Physics 40 (7) (2013) 072103. doi:http://dx.doi.org/10.1118/1.4810967

[20] M. Berger, J. Hubbell, Xcom: Photon cross sections on a personal computer., Report NBSIR873597, NIST, Gaithersburg MD20899.

[21] B. Walters, I. Kawrakow, D. Rogers, History by history statistical estimators in the beam code system, Medical Physics 29 (12) (2002) 2745-52. doi:https://doi.org/10.1118/1.1517611.

[22] I. Sechopoulos, E. S. M. Ali, A. Badal, A. Badano, J. M. Boone, I. S. Kyprianou, et al., Monte carlo reference data sets for imaging research: Executive summary of the report of aapm research committee task group 195, Medical Physics 42 (10) (2015) 5679-91. doi:http://dx.doi.org/ $10.1118 / 1.4928676$. 
[23] J. Siewerdsen, A. Waese, D. Moseley, S. Richard, D. Jaffray, Spektr: A computational tool for x-ray spectral analysis and imaging system optimization, monte carlo, Medical Physics 31 (11) (2004) 3057-67. doi:http://dx.doi.org/10.1118/1.1758350.

[24] A. C. Turner, D. Zhang, H. J. Kim, J. J. DeMarco, C. H. Cagnon, E. Angel, et al., A method to generate equivalent energy spectra and filtration models based onmeasurement for multidetector ct monte carlo dosimetry simulations, Medical Physics 36 (6) (2009) 2154-64. doi:http://dx. doi.org/10.1118/1.3117683

[25] M. Tapiovaaara, T. Siiskonen, PCXMC - A pc-based Monte Carlo program for calculating patient doses in medical x-ray Examinations, STUK - Radiation and Nuclear Safety Authority.

[26] A. Servomaa, M. Tapiovaara, Organ dose calculation in medical x ray examinations by the program pcxmc, Radiation Protection Dosimetry 80 (1-3) (1998) 213-9.

[27] X. G. Xu, An exponential growth of computational phantom research in radiation protection, imaging, and radiotherapy: a review of the fifty-year history, Physics in Medicine and Biology 59 (18) (2014) R233-R302. doi:10.1088/0031-9155/59/18/R233.

[28] C. Lee, W. Bolch, Age-dependent organ and effective dose coefficients for external photons: a comparison of stylized and voxel-based paediatric phantoms, Physics In Medicine And Biology 51 (18) (2006) 4663-88.

[29] R. Kramer, H. J. Khoury, J. W. Vieira, Comparison between effective doses for voxel-based and stylized exposure models from photon and electron irradiation, Physics in Medicine and Biology 50 (21) (2005) 5105-26.

[30] A. Dedulle, Dataset for validation of a monte carlo framework, Mendeley Data (2018). doi:http: //dx.doi.org/10.17632/fxj33nd36c.1.

[31] American Association of Physicists in Medicine, Electronic resources of the tg 195 report "monte carlo reference data sets for imaging research", https://www.aapm.org/pubs/reports/ report195.asp (Last accessed on 20/04/2018).

[32] A. Stratis, G. Zhang, X. Lopez-Rendon, R. Jacobs, R. Bogaerts, H. Bosmans, Customisation of a monte carlo dosimetry tool for dental cone-beam ct systems, Radiation Protection Dosimetry 169 (1-4) (2016) 378-85. doi:https://doi.org/10.1093/rpd/ncw024.

[33] D. Dance, S. Christofides, A. Maidment, I. McLean, K. Ng (Eds.), Diagnostic Radiology Physics: A Handbook for Teachers and Students, International Atomic Energy Agency, 2014. 
[34] X. Li, E. Samei, W. P. Segars, G. M. Sturgeon, J. G. Colsher, G. Toncheva, et al., Patient-specific radiation dose and cancer risk estimation in ct: Part i. development and validation of a monte carlo program, Medical Physics 38 (1) (2011) 397-407. doi:https://doi.org/10.1118/1.3515839.

[35] G. Jarry, J. DeMarco, U. Beifuss, C. Cagnon, M. McNitt-Gray, A monte carlo-based method to estimate radiation dose from spiral ct: from phantom testing to patient-specific models, Physics in Medicine and Biology 48 (16) (2003) 2645-63.

[36] P. Deak, M. van Straten, P. Shrimpton, M. Zankl, W. Kalender, Validation of a monte carlo tool for patient-specific dose simulations in multi-slice computed tomography, European Radiology 18 (4) (2008) 759 - 72. doi:https://doi.org/10.1007/s00330-007-0815-7.

[37] Dosimetry in Diagnostic Radiology: an International Code of Practice, International Atomic Energy Agency, 2007.

\section{Appendix A. Calibration equations}

Equation A.1 lists the numerical values of the curve fits for the calibration factor $\left(f_{c a l, U}\right)$ at a certain tube potential $(U)$ and related half-value layer $(H V L)$ for a given field of view at the position of the ionization chamber $\left(A_{F O V}[I C]\right)$. The curves are shown in Fig. 6

$$
\begin{aligned}
& f_{\text {cal }}=a \times A_{F O V}[I C] \\
& f_{c a l, 75 \mathrm{kV}, 2.86 \mathrm{mmAl}}=\left(2.31 \times 10^{8}\right) \frac{\text { hist }}{\mathrm{mAscm}^{2}} \times A_{F O V}[I C] \\
& f_{\text {cal }, 85 \mathrm{kV}, 3.11 \mathrm{mmAl}}=\left(3.26 \times 10^{8}\right) \frac{\mathrm{hist}}{\mathrm{mAs} \mathrm{cm}^{2}} \times A_{F O V}[I C] \\
& f_{c a l, 95 k V, 3.46 m m A l}=\left(4.15 \times 10^{8}\right) \frac{\text { hist }}{\mathrm{mAscm}^{2}} \times A_{F O V}[I C] \\
& f_{\text {cal }, 105 \mathrm{kV}, 4.37 \mathrm{mmAl}}=\left(5.37 \times 10^{8}\right) \frac{\text { hist }}{\mathrm{mAs} \mathrm{cm}^{2}} \times A_{F O V}[I C] \\
& f_{\text {cal }, 120 \mathrm{kV}, 5.01 \mathrm{mmAl}}=\left(7.56 \times 10^{8}\right) \frac{\text { hist }}{\mathrm{mAs} \mathrm{cm}^{2}} \times A_{F O V}[I C] \\
& f_{c a l, 125 \mathrm{kV}, 5.10 \mathrm{mmAl}}=\left(8.08 \times 10^{8}\right) \frac{\text { hist }}{\mathrm{mAscm}^{2}} \times A_{F O V}[I C]
\end{aligned}
$$

All curves had a correlation $\left(r^{2}\right)$ of 0.99 or above. 